A. B. Kharazishvili, Institute of Applied Mathematics of Tbilisi State University, University Street, 2, 380043 Tbilisi 43, Republic of Georgia.

e-mail: kharaz@saba.edu.ge

\title{
ON THE EXISTENCE OF NONMEASURABLE SUBGROUPS OF COMMUTATIVE GROUPS
}

\begin{abstract}
For a given commutative group $G$ equipped with a nonzero $\sigma$-finite diffused measure $\mu$, the question of the existence of a subgroup of $G$, nonmeasurable with respect to $\mu$, is discussed.
\end{abstract}

Let $G$ be a commutative group of cardinality of the continuum and let $\mu$ be a nonzero $\sigma$-finite diffused measure on $G$. In this paper, we consider the problem of the existence of a subgroup of $G$ which is nonmeasurable with respect to $\mu$.

We shall demonstrate, under the Continuum Hypothesis, that such a subgroup always exists. Moreover, Theorem 1 below contains a much stronger result stating that a nonmeasurable subgroup of $G$ can be found in some fixed countable family of subgroups of $G$.

We start with a well-known Banach-Kuratowski matrix (see [1]). Let us recall that if the Continuum Hypothesis holds, then, for every set $E$ of cardinality of the continuum, there exists a double family

$$
\left(E_{m, n}\right)_{m<\omega, n<\omega}
$$

of subsets of $E$, such that:

a) $E_{m, n} \subset E_{m, n+1}$ for all natural numbers $m$ and $n$;

b) $\cup\left\{E_{m, n}: n<\omega\right\}=E$ for any natural number $m$;

Key Words: commutative group, measure, nonmeasurable subgroup, Banach-Kuratowski matrix, Ulam matrix

Mathematical Reviews subject classification: 28A05, 28D05

Received by the editors June 4, 2000 
c) for each function $f: \omega \rightarrow \omega$, the set

$$
E_{0, f(0)} \cap E_{1, f(1)} \cap \cdots \cap E_{m, f(m)} \cap \cdots
$$

is at most countable.

The above-mentioned family $\left(E_{m, n}\right)_{m<\omega, n<\omega}$ is usually called a BanachKuratowski matrix over $E$. It was shown in [1] that, for every nonzero $\sigma$-finite diffused measure $\mu$ on $E$, at least one set $E_{m, n}$ is nonmeasurable with respect to $\mu$. Applying this classical result, we can establish the following statement.

Theorem 1. Assume that the Continuum Hypothesis is true, and let $G$ be a commutative group of cardinality of the continuum. Then there exists a countable family $\left(G_{i}\right)_{i \in I}$ of subgroups of $G$, such that, for any nonzero $\sigma$-finite diffused measure $\mu$ on $G$, at least one of the groups $G_{i}(i \in I)$ is nonmeasurable with respect to $\mu$.

Proof. According to the well-known theorem of the theory of commutative groups (see, e.g., [5]), the given group $G$ can be represented in the form

$$
G=\cup\left\{\Gamma_{k}: k<\omega\right\},
$$

where $\left(\Gamma_{k}\right)_{k<\omega}$ is an increasing countable family of subgroups of $G$ and each group $\Gamma_{k}$ is a direct sum of cyclic groups. In addition to this, we may suppose without loss of generality that all groups $\Gamma_{k}$ are of cardinality of the continuum. For every natural number $k$, we may write $\Gamma_{k}=\sum_{j \in J}\left[e_{k, j}\right]$ where $J$ is some set of cardinality of the continuum and $\left[e_{k, j}\right]$ denotes the cyclic group generated by $e_{k, j}$. We also write

$$
E_{k}=\left\{e_{k, j}: j \in J\right\} \text { for } k<\omega .
$$

Further, for any natural number $k$, let $\left(E_{k, m, n}\right)_{m<\omega, n<\omega}$ be a Banach-Kuratowski matrix over $E_{k}$ and let $\Gamma_{k, m, n}$ be the group generated by $E_{k, m, n}$. Then it is not hard to check that the family of groups $\left(\Gamma_{k, m, n}\right)_{m<\omega, n<\omega}$ is a BanachKuratowski matrix for the group $\Gamma_{k}$. Now, we put

$$
\left(G_{i}\right)_{i \in I}=\left\{\Gamma_{k}: k<\omega\right\} \cup\left\{\Gamma_{k, m, n}: k<\omega, m<\omega, n<\omega\right\},
$$

and we assert that the countable family $\left(G_{i}\right)_{i \in I}$ of subgroups of $G$ is the required one. Indeed, let $\mu$ be an arbitrary nonzero $\sigma$-finite diffused measure on $G$. Since every nonzero $\sigma$-finite measure is equivalent to a probability measure, we may assume without loss of generality that our $\mu$ is a probability measure. We have to show that at least one $G_{i}$ is nonmeasurable with respect to $\mu$. Suppose to the contrary that all $G_{i}(i \in I)$ are $\mu$-measurable. Then, 
taking into account the relation $G=\cup\left\{\Gamma_{k}: k<\omega\right\}$, we infer that there exists a natural number $r$ for which $\mu\left(\Gamma_{r}\right)>0$. In other words, $\mu$ is a nonzero finite diffused measure on the set $\Gamma_{r}$ and all subsets $\Gamma_{r, m, n}(m<\omega, n<\omega)$ of this set turn out to be $\mu$-measurable. But this is impossible since, as mentioned above, the family $\left(\Gamma_{r, m, n},\right)_{m<\omega, n<\omega}$ forms a Banach-Kuratowski matrix over $\Gamma_{r}$. The contradiction obtained ends the proof of Theorem 1.

Example. Denote by $\mathbb{R}$ the real line and consider an arbitrary nonzero $\sigma$ finite diffused measure $\nu$ on $\mathbb{R}$. If the Continuum Hypothesis holds, then, in view of the result presented above, there always exists a subgroup of the additive group $\mathbb{R}$, nonmeasurable with respect to $\nu$. Moreover, by applying an argument similar to the proof of Theorem 1, it can be shown, under $\mathbf{C H}$, that there always exists a vector subspace of $\mathbb{R}$ (over the rationals) which is nonmeasurable with respect to $\nu$ (cf. Remark 3 below).

In connection with Theorem 1, let us notice that some analogue of this theorem can be established by using Martin's Axiom instead of the Continuum Hypothesis. (Recall that Martin's Axiom is much weaker than $\mathbf{C H}$ and does not essentially restrict the size of the continuum.)

In our further considerations, we shall present an analogue of Theorem 1 for a commutative group $G$ whose cardinality is equal to the first uncountable cardinal $\omega_{1}$. Note that, in this case, the argument is more complicated and leads to a result in $\mathbf{Z F C}$ theory. Here the main role is played by a transfinite matrix of Ulam (see [6]).

We need several lemmas.

Lemma 1. Let $E$ be a set, $S$ be a $\sigma$-algebra of subsets of $E$ and let $I$ be $a \sigma$ ideal of subsets of $E$, such that $I \subset S$ and the pair $(S, I)$ satisfies the countable chain condition. Further, let $\left\{Z_{\alpha}: \alpha<\omega_{1}\right\}$ be an uncountable family of sets belonging to $S$, let $m>0$ be a fixed natural number and suppose that, for any m-element subset $D$ of $\omega_{1}$, the relation $\cap\left\{Z_{\alpha}: \alpha \in D\right\} \in I$ is fulfilled. Then there exists an uncountable subset $A$ of $\omega_{1}$ such that $Z_{\alpha} \in I$ for each ordinal $\alpha$ from $A$.

The proof of this lemma is not hard. It can be carried out by induction on $m$ (for details, see [3]). The case $m=2$ is, in fact, equivalent to the countable chain condition which says that any disjoint family of sets from $S \backslash I$ is at most countable. Also, using the induction on $m$, we are able to establish that the required set $A$ is actually co-countable in $\omega_{1}$ (and hence uncountable). In the sequel, only the uncountability of $A$ will be essentially utilized.

Let $Y$ be a set of cardinality $\omega_{1}$. Consider a double family $\left(Y_{n, \xi}\right)_{n<\omega, \xi<\omega_{1}}$ of subsets of $Y$. We shall say that this family is an admissible transfinite matrix for $Y$ if it possesses the following two properties: 
(1) for each ordinal number $\xi<\omega_{1}$, the partial family $\left(Y_{n, \xi}\right)_{n<\omega}$ is increasing by inclusion and $\cup\left\{Y_{n, \xi}: n<\omega\right\}=Y$;

(2) for each natural number $n$, there exists a natural number $m=m(n)$ such that, for any set $D \subset \omega_{1}$ with $\operatorname{card}(D)=m$, we have

$$
\operatorname{card}\left(\cap\left\{Y_{n, \xi}: \xi \in D\right\}\right) \leq \omega .
$$

Lemma 2. For any set $Y$ with $\operatorname{card}(Y)=\omega_{1}$, there exists an admissible transfinite matrix.

Proof. The argument is essentially based on the existence of an Ulam matrix over $Y$. Indeed, let $\left(X_{n, \xi}\right)_{n<\omega, \xi<\omega_{1}}$ be an arbitrary Ulam matrix for $Y$. Then we have:

(a) for each ordinal number $\xi<\omega_{1}$, the set $Y \backslash \cup\left\{X_{n, \xi}: n<\omega\right\}$ is at most countable;

(b) for each natural number $n$, the partial family $\left\{X_{n, \xi}: \xi<\omega_{1}\right\}$ is disjoint.

Define

$$
\begin{gathered}
\left\{x_{n, \xi}: n<\omega\right\}=Y \backslash \cup\left\{X_{n, \xi}: n<\omega\right\} ; \\
Y_{n, \xi}=\cup\left\{X_{k, \xi}: k \leq n\right\} \cup\left\{x_{k, \xi}: k \leq n\right\}
\end{gathered}
$$

for all $n<\omega$ and $\xi<\omega_{1}$. Then it is not hard to verify that the family $\left(Y_{n, \xi}\right)_{n<\omega, \xi<\omega_{1}}$ is an admissible matrix of subsets of $Y$. (Namely, for any natural number $n$, we may put $m(n)=n+2$.)

Lemma 3. Let $Y$ be a set of cardinality $\omega_{1}$ and let $\left(Y_{\alpha}\right)_{\alpha<\omega_{1}}$ be a family of subsets of $Y$. Then there exists a countably generated $\sigma$-algebra $S$ of subsets of $Y$, such that $\left\{Y_{\alpha}: \alpha<\omega_{1}\right\} \subset S$.

This lemma is well known and its proof is not difficult. It suffices to apply the so-called Sierpiński partition of the product set $\omega_{1} \times \omega_{1}$ (for details, see e.g. [4]).

Lemma 4. Let $Y$ be a set of cardinality $\omega_{1}$ and let $\left(Y_{n, \xi}\right)_{n<\omega, \xi<\omega_{1}}$ be an admissible transfinite matrix for $Y$. Then, for every nonzero $\sigma$-finite diffused measure $\mu$ on $Y$, at least one set $Y_{n, \xi}$ is nonmeasurable with respect to $\mu$.

Note that Lemma 4 follows easily from Lemma 1 (cf. also [3]).

Lemma 5. Let $\Gamma$ be a commutative group representable in the form of a direct sum $\Gamma=\sum_{\zeta<\omega_{1}}\left[e_{\zeta}\right]$, where $\left(e_{\zeta}\right)_{\zeta<\omega_{1}}$ is an injective family of elements of $\Gamma$, and let $\left(E_{n, \xi}\right)_{n<\omega, \xi<\omega_{1}}$ be an admissible transfinite matrix over the set $E=\left\{e_{\zeta}: \zeta<\omega_{1}\right\}$. For each subset $F$ of $E$, denote by $[F]$ the group generated by $F$. Then the family of groups $\left(\left[E_{n, \xi}\right]\right)_{n<\omega, \xi<\omega_{1}}$ is an admissible transfinite matrix over $\Gamma$. 
The proof of Lemma 5 is reduced to a direct verification.

Now, we can formulate and prove the following statement.

Theorem 2. Let $G$ be any commutative group of cardinality $\omega_{1}$. Then there exists a countable family $\left(G_{i}\right)_{i \in I}$ of subgroups of $G$, such that, for every nonzero $\sigma$-finite diffused measure $\mu$ on $G$, at least one group $G_{i}$ is nonmeasurable with respect to $\mu$.

Proof. As we know, our group $G$ can be represented in the form

$$
G=\cup\left\{\Gamma_{k}: k<\omega\right\}
$$

where $\left(\Gamma_{k}\right)_{k<\omega}$ is an increasing family of subgroups of $G$ and each $\Gamma_{k}$ is a direct sum of cyclic groups. We may assume without loss of generality that all $\Gamma_{k}$ are of cardinality $\omega_{1}$. So we may write $\Gamma_{k}=\sum_{\zeta<\omega_{1}}\left[e_{k, \zeta}\right]$. For $k<\omega$ put $E_{k}=\left\{e_{k, \zeta}: \zeta<\omega_{1}\right\}$ and let $\left(E_{k, n, \xi}\right)_{n<\omega, \xi<\omega_{1}}$ be an admissible transfinite matrix over $E_{k}$. (This matrix exists according to Lemma 2.) Further, applying Lemma 3 , we see that there exists a countable family $S_{k}$ of subsets of $E_{k}$, such that

$$
\left\{E_{k, n, \xi}: n<\omega, \xi<\omega_{1}\right\} \subset \sigma\left(S_{k}\right)
$$

where $\sigma\left(S_{k}\right)$ denotes the $\sigma$-algebra of sets, generated by $S_{k}$. Clearly, we may suppose that $S_{k}$ is an algebra of sets. Consequently, the $\sigma$-algebra $\sigma\left(S_{k}\right)$ coincides with the monotone class generated by $S_{k}$ (see, e.g., [2]).

Now, we put

$$
\left(G_{i}\right)_{i \in I}=\left\{\Gamma_{k}: k<\omega\right\} \cup\left\{[Z]: Z \in \cup_{k<\omega} S_{k}\right\},
$$

and we assert that the family of groups $\left(G_{i}\right)_{i \in I}$ is the required one. To see this, take any nonzero $\sigma$-finite diffused measure $\mu$ on $G$ and suppose to the contrary that all groups $G_{i}(i \in I)$ are $\mu$-measurable. Then, for some $r<\omega$, we must have $\mu\left(\Gamma_{r}\right)>0$. Consider the countable family of groups

$$
P_{r}=\left\{[Z]: Z \in S_{r}\right\} .
$$

An easy transfinite induction shows that all groups $\left[E_{r, n, \xi}\right]$ for $n<\omega, \xi<\omega_{1}$ belong to the monotone class generated by $P_{r}$. But, according to our assumption, all sets from $P_{r}$ are $\mu$-measurable. Therefore, all groups $\left[E_{r, n, \xi}\right]$ must be $\mu$-measurable, too, which contradicts Lemmas 4 and 5 . The contradiction obtained finishes the proof of Theorem 2.

Remark 1. Obviously, Theorem 1 follows from Theorem 2.

Remark 2. For noncommutative groups of cardinality $\omega_{1}$, Theorem 2 fails to hold (cf. [4], p. 87). 
Remark 3. It is easy to see that direct analogues of Theorems 1 and 2 are valid for vector spaces (e.g. over the field $\mathbb{Q}$ of all rationals) instead of commutative groups. In particular, if $V$ is a vector space over $\mathbb{Q}$ with $\operatorname{card}(V)=\omega_{1}$, then there exists a countable family $\left(V_{i}\right)_{i \in I}$ of vector subspaces of $V$, such that, for any nonzero $\sigma$-finite diffused measure $\mu$ on $V$, at least one $V_{i}$ is nonmeasurable with respect to $\mu$.

It would be interesting to extend Theorems 1 and 2 (respectively, their above-mentioned analogues) to a wider class of uncountable commutative groups $G$ (respectively, to a wider class of uncountable vector spaces $V$ ). Namely, the following question seems to be of some interest.

Let $G$ be a commutative group whose cardinality is not real-valued measurable, and let $\mu$ be a nonzero $\sigma$-finite diffused measure on $G$. Does there exist a subgroup of $G$ nonmeasurable with respect to $\mu$ ?

\section{References}

[1] S. Banach, K. Kuratowski, Sur une generalisation du probleme de la mesure, Fund. Math., 14, (1929), 127-131.

[2] P. R. Halmos, Measure Theory, D. Van Nostrand, New York, 1950.

[3] A. B. Kharazishvili, Some remarks on additive properties of invariant $\sigma$ ideals on the real line, Real Analysis Exchange, 21, no. 2, (1995-1996), $715-724$.

[4] A. B. Kharazishvili, Transformation Groups and Invariant Measures, World Scientific Publishing Co., Singapore, 1998.

[5] A. G. Kurosh, The Theory of Groups, Izd. Nauka, Moscow, 1967 (in Russian).

[6] S. Ulam, Zur Masstheorie in der allgemeinen Mengenlehre, Fund. Math., 16, (1930), 140-150. 